\title{
Model refinements and experimental testing of highly flexible piezoelectric energy harvesters
}

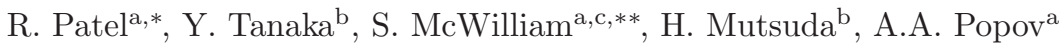 \\ ${ }^{a}$ Materials, Mechanics and Structures Research Division, Faculty of Engineering, \\ University of Nottingham, Nottingham, NG7 2RD, UK \\ ${ }^{b}$ Division of Energy and Environmental Engineering, Hiroshima University, \\ 1-4-1 Kagamiyama, Higashi-Hiroshima, Japan \\ ${ }^{c}$ Materials, Mechanics and Structures Research Division, Faculty of Engineering, \\ University of Nottingham Malaysia Campus, 43500 Semenyih, Malaysia
}

\begin{abstract}
This paper addresses limitations to existing analytical models for piezoelectric energy harvesters. The presented model is targeted at predicting behaviours of highly flexible piezoelectric devices (FPEDs) and includes high orders of substrate and piezoelectric material non-linearity, geometric non-linearity, and additionally the effects of both self-weight and pre-stress. Validation through experimental testing is provided.

The influence of self-weight on vibratory dynamics becomes important in FPEDs due to both material composition and dimension. The developed model facilitates the simulation of FPED performance mounted at specified angles to the horizontal. In one study, for a FPED of $120 \mathrm{~mm}$ in length, the resonant frequency changed by over $30 \%$ with mounting angle. Consideration of mounting orientation is advised as self-weight increases damping and significantly lowers FPED performance - over a $50 \%$ reduction in one presented case.
\end{abstract}

Keywords:

Energy harvesting, Flexible device, Piezoelectric beam, Self-weight, Pre-stress, Initial curvature

\section{Introduction}

Energy harvesting is a topic of global appeal, and is receiving growing interest in terms of both research and practical application possibilities. In essence, the term refers to the conversion of wasted ambient energy into useful energy,

\footnotetext{
*Corresponding author

** Principal corresponding author

Email addresses: Rupesh.Patel@nottingham.ac.uk (R. Patel), yoshi@naoe.hiroshima-u.ac.jp (Y. Tanaka), Stewart.McWilliam@nottingham.ac.uk (S. McWilliam), mutsuda@hiroshima-u.ac.jp (H. Mutsuda), Atanas.Popov@nottingham.ac.uk (A.A. Popov)
} 
often electrical. The focus here is on wasted kinetic energy, where transduction methods such as piezoelectricity and electromagnetism are utilised for energy capture. Amongst researchers the consensus is that piezoelectric transduction offers superior conversion efficiencies [1] and this mechanism is adopted in this work. The prime utilisation of energy generated by such means is to power miniature wireless sensors, e.g. tyre pressure monitoring sensors or temperature/humidity sensors. However, an investigation of using such technology to extract power on a significantly larger scale, i.e. $\mathrm{kWs}$ from environments such as oceans or wind fields, is currently under way. To this end, Mutsuda et al. 22 and Tanaka et al. 3. have been developing the concept of employing highly flexible piezoelectric devices (FPEDs), cantilever in form, comprised of silicone rubber (Si) and polyvinylidene fluoride (PVDF) thin films. The overarching vision is to create 'offshore renewable energy generation farms' by combining several concepts (e.g. wave motion FPEDs and wind turbines). This proposition is one solution to the inexorable rise in global energy demand whilst reducing reliance on fossil fuels and slowing global warming. The nature of FPEDs makes them ideal for the aforementioned target application environments due to their inherently low fundamental frequencies.

Early analytical modelling of cantilever piezoelectric harvesters predominantly assumed linear behaviour. Sodano et al. [4] used the Rayleigh-Ritz procedure for estimating the power output from a cantilever mounted piezoelectric generator. Ertuk and Inman [5] followed this by developing a distributedparameter electromechanical model for energy harvesters. Patel et al. [6] extended the model by accurately incorporating effects of non-uniform beams created by altering coverage of the piezoelectric layer. Utilisation of the model showed improvements in performance are achievable by optimising piezoelectric length, with experimental data providing model validity. More recently the importance of using non-linear models for theoretical performance predictions has come to light [7-10]. Non-linearity in piezoelectric materials [11], in addition to geometric non-linearity created in structures undergoing large deformations, necessitates the use of non-linear models. Stanton's early work [7] saw the development of a non-linear model which considered piezoelectric material non-linearity in the form of higher order terms in constitutive equations. This was later extended in 9], where tip mass effects and further higher order terms where included to increase model robustness. For the common cantilever harvester design it is recognised and documented that performance drops off significantly as a result of mismatch between resonant and excitation frequency. It is therefore important to be able to predict how non-linear behaviour affects the frequency response of devices to ensure harvesters are designed to maximise performance.

The devices considered by Mutsuda et al. 2, and Tanaka et al. 3 are made from Si and PVDF layers and are highly flexible. As such they are likely to experience geometric non-linearity and exhibit high levels of material nonlinearity. Modelling performed by Patel et al. in 10 is well suited to analysing this case as it incorporates the effects of substrate, piezoelectric and geometric non-linearity. Serving as a basis, alterations will be made in line with Stanton 
et al. 9] to include higher orders of odd power non-linearity in material constitutive equations; recognised as essential for increasing the model validity range. Differentiating from previous work, several additional key effects are also important when considering FPEDs. Firstly, self-weight influences vibratory dynamics and can be observed in the behaviour of long slender beams whether mounted horizontally or vertically. Vertical cantilevers oriented downwards stiffen due to gravitational forces with opposing effects present in beam oriented upwards against the acting direction of gravity [12. Self-weight has previous been overlooked in work related to energy harvesters, for example in Hobeck and Inman [13] and Xie et al. [14, who both considered vertically oriented devices. In contrast, Friswell et al. [15] performed work on vertically oriented devices and integrated self-weight effects however modelling excluded material non-linearity. The model developed in this paper will incorporate the influence of self-weight on FPEDs mounted at angles ranging from $-90^{\circ}$ to $+90^{\circ}$ to the horizontal. Pre-stress is the second key issue to influence dynamical behaviour of FPEDs. Residual stresses arise in PVDF thin films during manufacture as published by Oh et al. [16, in addition to those created during adhesive curing via chemical shrinkage [17. Modelling and predicting residual stress effects is essential to accurately simulate the dynamics and electrical performance of FPEDs.

This paper details theoretical model refinements to an existing non-linear model for piezoelectric energy harvesters, in terms of (i) higher order odd power material non-linearity, (ii) sample self-weight and (iii) inherent pre-stress in samples. Through experimental testing of devices manufactured 'in-house' the verification of these extensions and model validation is provided. The paper is laid out as follows. Firstly, a recap of previous non-linear modelling is detailed with modifications made to accommodate higher order odd power material nonlinearity. Secondly, data from experimental testing is provided to demonstrate the necessity of including self-weight and pre-stress and the effects they have on energy harvesting. In this section theoretical model derivations of the effects will also be presented. In the second part of the paper, attention is turned to validation. Firstly, unknown material properties, including coefficients of material non-linearity, will be obtained through static and dynamic testing. Subsequently, model extensions relating to self-weight and pre-stress are validated individually. Additionally, the analytical model is used to highlight the importance of these effects in the prediction of FPED behaviour.

\section{Modelling of a highly flexible piezoelectric device}

In this section the development and expansion of an analytical model is presented. The model is targeted at predicting the electrical performance and dynamical behaviour of FPEDs. Non-linear work previously conducted by Patel et al. [10] and Stanton et al. 9] forms the basis for the model core.

A unimorph energy harvester schematic with the dimensional and directional notion used throughout is shown in Figure 1. $x_{1}$ represents the distance of the piezoelectric layer from the clamped end, and $x_{2}$ is the length of the piezoelectric layer. The Newtonian inertial co-ordinate system is represented by $(x, y, z)$ 


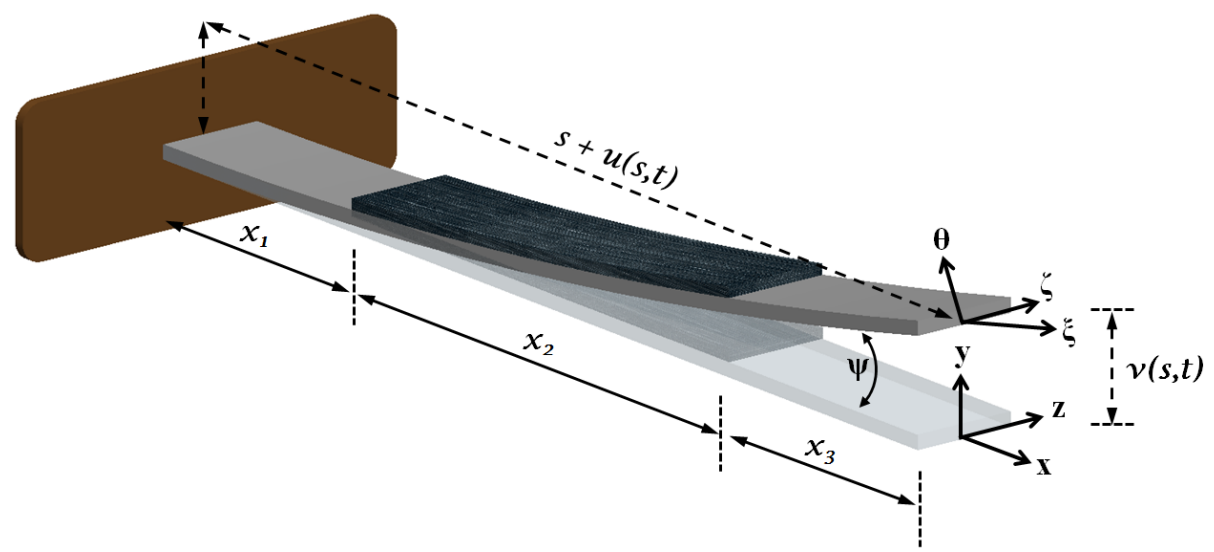

Figure 1: Dimensional and directional notation used through this paper.

and the local co-ordinate system by $(\xi, \theta, \zeta)$. The beam deforms as shown in Figure 1 such that the transverse and longitudinal time-varying deformations are represented by $v(s, t)$ and $u(s, t)$ respectively. For the purpose of compactness, in all subsequent equations and text the dependencies of $v(s, t)$ and $u(s, t)$ on $s$ and $t$ will be omitted.

\subsection{Recap of previous model including various non-linearities}

Previous work on the non-linear modelling of piezoelectric vibrational energy harvesters was presented by Patel et al. 10. Included were both:

- material non-linearity in the form of a singular higher order term in each material constitutive equation.

- geometric non-linearity by assuming beam inextensibility.

Note the inclusion of material non-linearity in the substrate layer which will be important here as a result of using silicone rubber $(\mathrm{Si})$ for the support layer.

From [10, the constitutive equation for the substrate material is:

$$
\sigma_{11}^{s}=E_{s} \varepsilon_{11}^{s}+\frac{\mu_{s 1}}{2}\left(\varepsilon_{11}^{s}\right)^{2},
$$

where $\sigma_{11}$ is the axial stress, $\varepsilon_{11}$ is the axial strain and superscript $s$ refers to the substrate layer. The coefficient of material non-linearity for the substrate is assigned $\mu_{s 1}$, and $E_{s}$ is the substrate material Young's modulus.

Likewise from [10], the constitutive equation for the piezoelectric material is:

$$
\begin{aligned}
\sigma_{11}^{p} & =E_{p} \varepsilon_{11}^{p}+\frac{\mu_{1}}{2}\left(\varepsilon_{11}^{p}\right)^{2}-E_{p} d_{31} E_{f}-\mu_{2} \varepsilon_{11}^{p} E_{f}, \\
D_{3} & =E_{p} d_{31} \varepsilon_{11}^{p}+\frac{\mu_{2}}{2}\left(\varepsilon_{11}^{p}\right)^{2}+\varepsilon_{33} E_{f},
\end{aligned}
$$


where superscript $p$ refers to the piezoelectric layer, $E_{p}$ is the piezoelectric material Young's modulus, and $E_{f}$ the electric field strength. $d_{31}$ is a piezoelectric material constant, and $\varepsilon_{33}$ is the material permittivity. Constants $\mu_{1}$ and $\mu_{2}$ represent non-linearity, and are both specific and unique to each 'batch' of piezoelectric material.

However, as detailed by Stanton et al. in [9, higher order terms with odd powers of strain are required in constitutive equations for non-linear effects in bimorph compositions to remain. More importantly, the higher order terms are essential to better capture dynamic behaviour across a wider span of excitation conditions. For these reasons the above constitutive relations, Equations (1)-(3), are modified to the following quintic functions:

$$
\begin{aligned}
& \sigma_{11}^{s}=E_{s} \varepsilon_{11}^{s}+\mu_{s 1}\left(\varepsilon_{11}^{s}\right)^{3}+\mu_{s 2}\left(\varepsilon_{11}^{s}\right)^{5}, \\
& \sigma_{11}^{p}=E_{p} \varepsilon_{11}^{p}+\mu_{p 1}\left(\varepsilon_{11}^{p}\right)^{3}+\mu_{p 2}\left(\varepsilon_{11}^{p}\right)^{5}-E_{p} d_{31} E_{f}-\mu_{p 3} \varepsilon_{11}^{p} E_{f},
\end{aligned}
$$

and,

$$
D_{3}=E_{p} d_{31} \varepsilon_{11}^{p}+\mu_{p 3}\left(\varepsilon_{11}^{p}\right)^{2}+\varepsilon_{33} E_{f} .
$$

Kinetic and potential energy expressions are initially obtained for each of the three beam segments shown in Figure 1 by using Equations (4)-(6).

Kinetic energy:

$$
T=\frac{1}{2} \int_{0}^{L} m(s)\left[\left(\int_{0}^{s} v^{\prime} \dot{v}^{\prime}\right)^{2}+\dot{v}^{2}\right] \mathrm{d} s,
$$

Potential energy:

$$
\begin{aligned}
U=\frac{1}{2} \int_{0}^{L} & \left\{\left(K_{1}(s)-K_{5}(s) V(t)\right)\left(\left(v^{\prime \prime}\right)^{2}+\left(v^{\prime \prime} v^{\prime}\right)^{2}\right)+K_{2}(s)\left(v^{\prime \prime}\right)^{4}\right. \\
& \left.+K_{3}(s)\left(v^{\prime \prime}\right)^{6}-K_{4}(s)\left(v^{\prime \prime}+\frac{1}{2} v^{\prime \prime}\left(v^{\prime}\right)^{2}\right) V(t)-K_{6}(s) V^{2}(t)\right\} \mathrm{d} s .
\end{aligned}
$$

where $m(s)$ is mass-per-unit-length of the FPED and constants $K_{1}$ to $K_{6}$ are defined in Appendix A. Note how the inextensibility condition, Equation (9), has been used to relate the longitudinal slope to the transverse slope.

$$
u^{\prime} \approx-\frac{1}{2}\left(v^{\prime}\right)^{2} .
$$

A sub-model utilising the transfer matrix method allows for design optimisations of piezoelectric material coverage by providing natural frequencies and linear vibrational mode shapes of segmented beams based on classical beam theory. This information is used with Galerkin's method and extended Hamilton's principle to obtain non-linear equations of motion from the energy integrals. To avoid procedural repetitiveness, detailed derivation steps are omitted here and the reader is referred to Patel 18 for general step-by-step procedures; albeit for 
a case with lower orders of non-linearity. Final expressions using the modified constitutive equations, Equations (4)-(6), are provided here:

$$
\begin{aligned}
\ddot{\eta}_{r}(t) & +2 \gamma_{r} \omega_{r} \dot{\eta}_{r}(t)+\omega_{r}^{2} \eta_{r}(t)+\left(C_{n 1}^{r}+C_{n 2}^{r}\right) \eta_{r}^{3}(t)+C_{n 3}^{r} \eta_{r}^{5}(t)+C_{n 4}^{r} \ddot{\eta}_{r}(t) \eta_{r}^{2}(t) \\
& +C_{n 5}^{r} \dot{\eta}_{r}^{2}(t) \eta_{r}(t)-C_{n 6}^{r} V(t)-C_{n 7}^{r} \eta_{r}(t) V(t) \\
& +C_{n 8}^{r} \eta_{r}^{2}(t) V(t)-C_{n 9}^{r} \eta_{r}^{3}(t) V(t)=C_{n 10}^{r} \ddot{w}_{b}(t)
\end{aligned}
$$

and

$$
\begin{aligned}
C_{n 11}^{r} \eta_{r}(t) \dot{\eta}_{r}(t)+ & C_{n 12}^{r} \eta_{r}^{3}(t) \dot{\eta}_{r}(t)+C_{n 13}^{r} \dot{\eta}_{r}(t)+C_{n 14}^{r} \eta_{r}^{2}(t) \dot{\eta}_{r}(t) \\
& +C_{n 15}^{r} \dot{V}(t)+\frac{V(t)}{R_{l}}=0 .
\end{aligned}
$$

where $\gamma_{r}$ and $\omega_{r}$ represent mechanical damping ratio and natural frequency of the $r^{\text {th }}$ mode. $\ddot{w}_{b}(t)$ is the base acceleration, $\eta_{r}(t)$ is the time dependant generalised co-ordinates, and $V(t)$ is the voltage generated across a resistor, $R_{l}$. $C_{n 1}^{r}$ through $C_{n 15}^{r}$ are time-independent constants and can be found in Appendix B. Besides changes to the time independent constants, the differences between Equation 10] and that found in [18] are (i) the inclusion of a $\eta_{r}^{5}$ term and (ii) the replacement of the $\eta_{r}^{2}$ term by a $\eta_{r}^{3}$ term. Equations 10 and (11) are single mode approximations solved numerically using ordinary differential equation (ODE) solvers in MATLAB ${ }^{\circledR}$, through the SimulinK ${ }^{\circledR}$ interface [19], with data recorded once steady-state conditions have been achieved. During experimental work and model validation, only excitation around the fundamental frequency is of interest. For this reason, during analytical model use, Equations (10) and (11) will be solved for $r=1$ only. This section has developed a model based on Stanton et al. [9] and Patel et al. [10. The following sections will show extensions to this base model in relation to phenomenon particularly important to highly flexible devices.

\subsection{Inclusion of self-weight}

The inclusion of self-weight in the analytical modelling of FPEDs was found to be essential from preliminary experimental work. Figure 2 provides frequency responses when testing a device in three orientations. In the horizontal orientation the device was mounted and tested with its thickness direction parallel to the horizontal thereby minimising gravitational effects, see Figure 3 . The sample comprises of a $3 \mathrm{~mm}$ thick Si substrate and $0.08 \mathrm{~mm}$ thick PVDF thin film acting as the active piezoelectric material. Bonding is realised through the application of a silicon-based adhesive in order to provide both flexibility and adequate cohesion between layers. The device has a width and overhang length of $12 \mathrm{~mm}$ and $100 \pm 1 \mathrm{~mm}$, respectively.

A Data Physics GW-V4 electromagnetic shaker provides base excitation with a Stanford Research Systems SR785 dynamic signal analyser outputting a har-

monic signal to the shaker via a standard amplifier. One input channel of the analyser is used to monitor base acceleration from a PCB Piezotronic accelerometer (model number - 352C23), with feedback control ensuring constant 


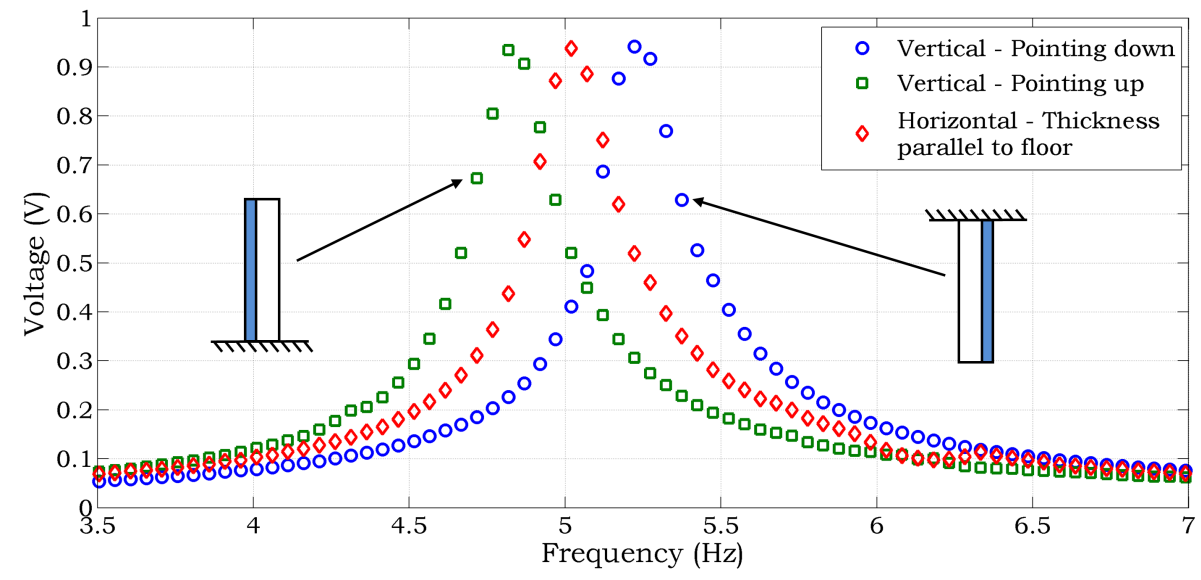

Figure 2: Experimental data demonstrating influence of beam orientation on the frequency response of a FPED. Horizontal configuration with thickness direction parallel to the floor is used as a control whereby self-weight effects are minimised.

acceleration to $\pm 0.1 \mathrm{~dB}$ during frequency sweeping. The second channel measures either point velocity, obtained from a PolyTec OFV-055 laser vibrometer, or voltage generated by the FPED. Throughout the paper, base acceleration is consistently applied parallel to the sample thickness direction at the root; in this current test a magnitude of $1 \mathrm{~ms}^{-2}$ is applied. Due to low resonant frequencies of FPEDs, settle times of over $5 \mathrm{~s}$ for each excitation frequency are used to avoid data recording from the transient phase. In all cases the voltage is measured in open circuit conditions, i.e. electrodes of the PVDF layer are connected to the analyser directly through a passive oscilloscope probe.

Results in Figure 2 indicate the device mounted horizontally possessed a resonant frequency of $5.02 \mathrm{~Hz}$. For vertical orientations pointing downwards and upwards this changed to $5.24 \mathrm{~Hz}$ and $4.83 \mathrm{~Hz}$ respectively. The percentage difference between the two extreme orientations is $8.5 \%$. More importantly, due to high quality factors, miss-predications of resonant frequency during design can lead to the device operating at $50 \%$ less-than-peak performance from self-weight effects alone. On a side note, one of the key challenges faced in vibration energy harvesting is the heavy dependency of performance on excitation frequency however this is not the focus here. For more information on this matter the reader is referred to a review by Tang et al. [20] where details and comparisons on techniques such as (i) preloading, and (ii) introducing non-linearity through magnets, are presented as attempts to reduce this dependency.

In what follows, self-weight is included in the analytical modelling. Firstly, the transfer matrix (TM) used to obtain natural frequencies and mode shapes for segmented beams requires modification to accommodate gravitational loading. The form of the TM for cases including forcing is readily available in the literature, see for example [21, and is not repeated here. In essence the TM changes from a previously utilised $4 \times 4$ matrix to a $5 \times 5$ matrix, with the 5 th 


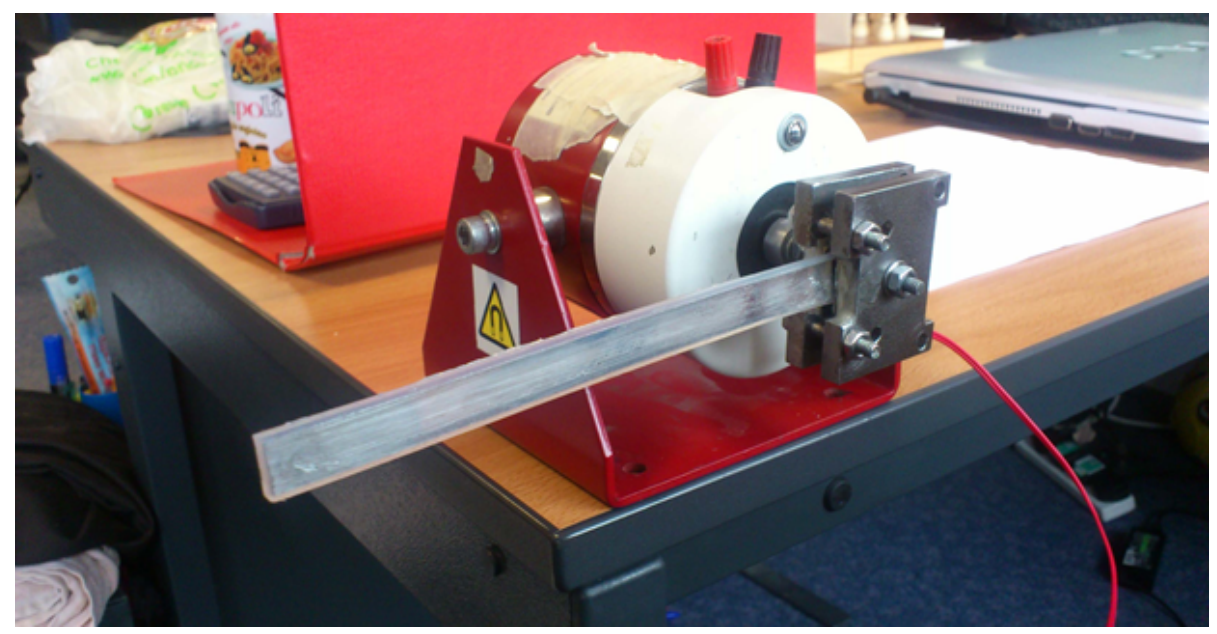

Figure 3: Horizontal orientation with thickness parallel to the floor.

column representing the distributed force; consequently, beam mode shapes are affected through this constant distributed load.

Secondly, and more importantly, gravitational loading gives rise to additional energy terms in the equation of motion, Equation (10). During experimentation and modelling of gravitational loading, the beam will mostly be oriented at angles about the z-axis in Figure1. A schematic diagram representing deformations of an arbitrarily orientated harvester (angle, $\theta$, to the horizontal, positive in the anticlockwise direction) is shown in Figure 4. To determine the influence of self-weight on the beam it is necessary to consider the weight force acting on an element as shown in Figure 4 . The gravitational potential energy for a beam segment of length $\mathrm{d} s$ is given by:

$$
m g(s \sin \theta+v \cos \theta+u \sin \theta) \mathrm{d} s,
$$

where $g$ is the gravitational constant, $9.81 \mathrm{~ms}^{-2}$. In what follows the $m g \sin \theta$ term, which accounts for the rigid body gravitation potential energy, is neglected. Consequently the gravitational potential energy due to elastic deformation for the entire beam is given by:

$$
U_{g}=m g \int_{0}^{L}(v \cos \theta+u \sin \theta) \mathrm{d} s,
$$

where $L$ is the total beam length. As the beam is assumed inextensible, to make use of Equation (9), the second term in Equation 13 must be integrated by parts as shown below:

$$
m g \sin \theta \int_{0}^{L} u \mathrm{~d} s=m g \sin \theta\left([u s]_{0}^{L}-\int_{0}^{L} u^{\prime} s \mathrm{~d} s\right) .
$$




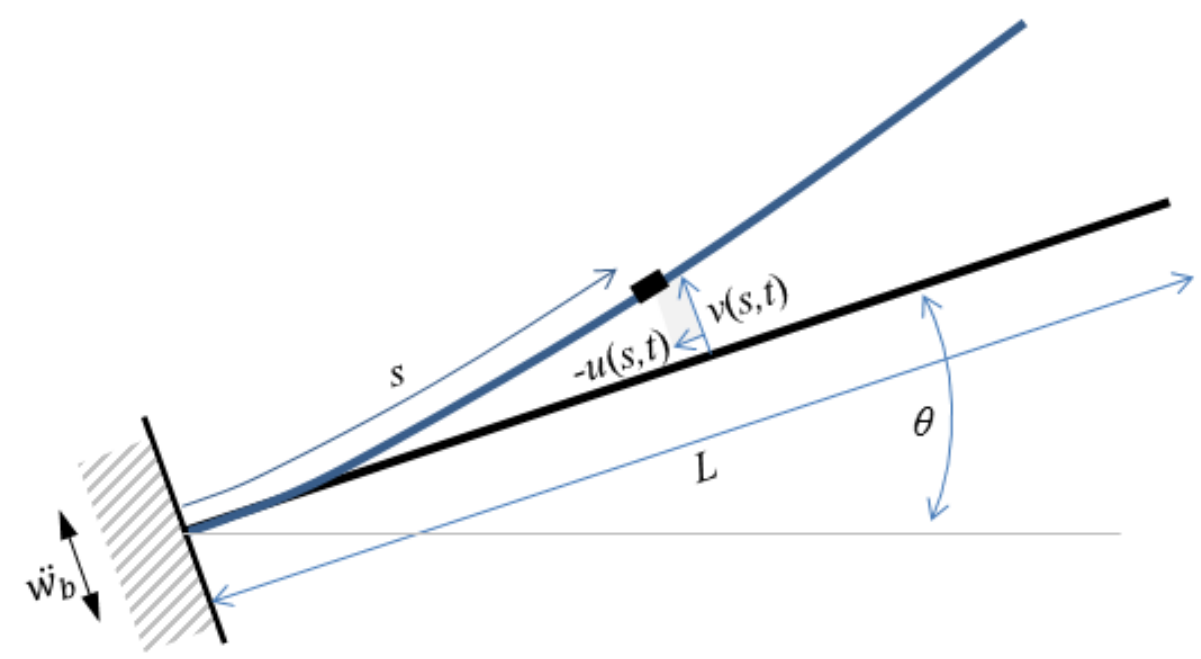

Figure 4: Schematic of the orientated harvester with dimension and deformation symbols indicated.

where $\left({ }^{\prime}\right)$ donates the derivative with respect to arc length, $s$. Noting that $u=0$ at $s=0$ and $u=\int_{0}^{L} u^{\prime} \mathrm{d} s$ at $s=L$, Equation 14 can be expressed as:

$$
-m g \sin \theta \int_{0}^{L}(L-s) u^{\prime} s \mathrm{~d} s .
$$

Finally, using the inextensibility relationship on Equation 15 and substituting into Equation (13) one obtains the following expression for total gravitational potential energy due to elastic deformation:

$$
U_{g}=\int_{0}^{L} m g\left(v \cos \theta-\frac{1}{2}\left(v^{\prime}\right)^{2}(L-s) \sin \theta\right) \mathrm{d} s .
$$

An additional term must also be introduced whose importance will be highlighted in the results section later, see Section 3.3.4. Initial deformation resulting from self-weight creates longitudinal tension in the beam and an expression for this is readily obtained using the procedure outlined above. The constant beam orientation angle, $\theta$, is replaced by the initial slope of the cantilever, $v_{i}^{\prime}$; tension in the sample resulting from initial deformation therefore gives rise to:

$$
U_{o}=-\int_{0}^{L} m g\left(\frac{1}{2}\left(v^{\prime}\right)^{2}(L-s) v_{i}^{\prime}\right) \mathrm{d} s .
$$

Equations (16) and (17) are added to the potential energy expression, Equation (8), before applying the extended Hamilton's principle and utilising the calculus of variations. This leads to the following additional terms:

$$
K_{7}(s)+\left(K_{8}(s) v^{\prime}\right)^{\prime}
$$


where $K_{7}(s)$ and $K_{8}(s)$ are expressed by:

$$
\begin{array}{r}
K_{7}(s)=m(s) g \cos \theta, \\
K_{8}(s)=m(s) g(L-s)\left(\sin \theta+v_{i}^{\prime}\right) .
\end{array}
$$

The adoption of the Bubnov-Galerkin method, where $v$ is expressed as an infinite sum of products of normalised eigenvectors, $W_{r}(s)$, and time-dependant generalised coordinates, $\eta_{r}(t)$, followed with multiplying through by $W_{r}(s)$ and integrating over $L$, yields:

$$
\int_{0}^{L} K_{7}(s) W_{r}(s) \mathrm{d} s+\eta(t) \int_{0}^{L}\left(K_{8}(s) W_{r}^{\prime}(s)\right)^{\prime} W_{r}(s) \mathrm{d} s .
$$

This expression can be incorporated into Equation 10 to give:

$$
\begin{aligned}
\ddot{\eta}_{r}(t) & +2 \gamma_{r} \omega_{r} \dot{\eta}_{r}(t)+\left(\omega_{r}^{2}+\int_{0}^{L}\left(K_{8}(s) W_{r}^{\prime}(s)\right)^{\prime} W_{r}(s) \mathrm{d} s\right) \eta_{r}(t)+\left(C_{n 1}^{r}+C_{n 2}^{r}\right) \eta_{r}^{3}(t) \\
& +C_{n 3}^{r} \eta_{r}^{5}(t)+C_{n 4}^{r} \ddot{\eta}_{r}(t) \eta_{r}^{2}(t)+C_{n 5}^{r} \dot{\eta}_{r}^{2}(t) \eta_{r}(t)-C_{n 6}^{r} V(t)-C_{n 7}^{r} \eta_{r}(t) V(t) \\
& +C_{n 8}^{r} \eta_{r}^{2}(t) V(t)-C_{n 9}^{r} \eta_{r}^{3}(t) V(t)=C_{n 10}^{r} \ddot{w}_{b}(t)+\int_{0}^{L} K_{7}(s) W_{r}(s) \mathrm{d} s .
\end{aligned}
$$

The additional terms in Equation (22) indicate that self-weight influences both the stiffness of the device and the forcing magnitude. It is worth mentioning the importance of utilising the added effects from self-weight in conjunction with a non-linear model. Experimental data provided in Section 3.3.3, i.e. Figure 13 . indicates a highly non-linear system dominated by material non-linearity causing peaks to turn towards the lower frequency spectrum for increasing acceleration levels.

\subsection{Inclusion of pre-stress}

Another important phenomenon observed from experimental testing is the presence and influence of pre-stress. Residual stresses can occur naturally in PVDF thin films as a result of the manufacturing process [16 and also in the adhesive layer as a result of shrinkage during the curing process 17. Figure 5 illustrates the effects of pre-stress by reversing the orientation of a horizontally clamped device. Due to misalignment between neutral axis location and prestress location, a moment is experienced by samples about the neutral axis. This either amplifies or diminishes initial deflection from self-weight depending on device orientation and composition.

Experimental data in Figure 6 shows the effect of pre-stress on the dynamical behaviour of FPEDs. Although the peak voltage of the device is unaffected, a shift in resonant frequency is experienced. In this particular example a frequency shift of $2.08 \%$ was recorded. With the exception of length, the device under examination is identical to that tested in Section 2.2. The overhang length in this case was measured at $70 \pm 1 \mathrm{~mm}$. 

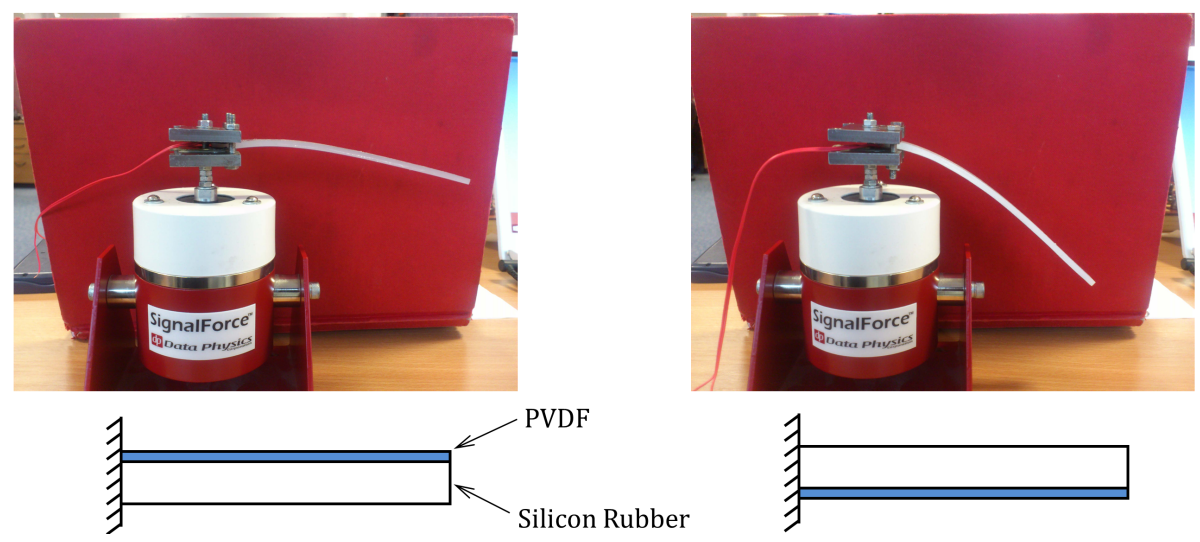

Figure 5: Static visualisation of the influence of pre-stress in an asymmetric energy harvester.

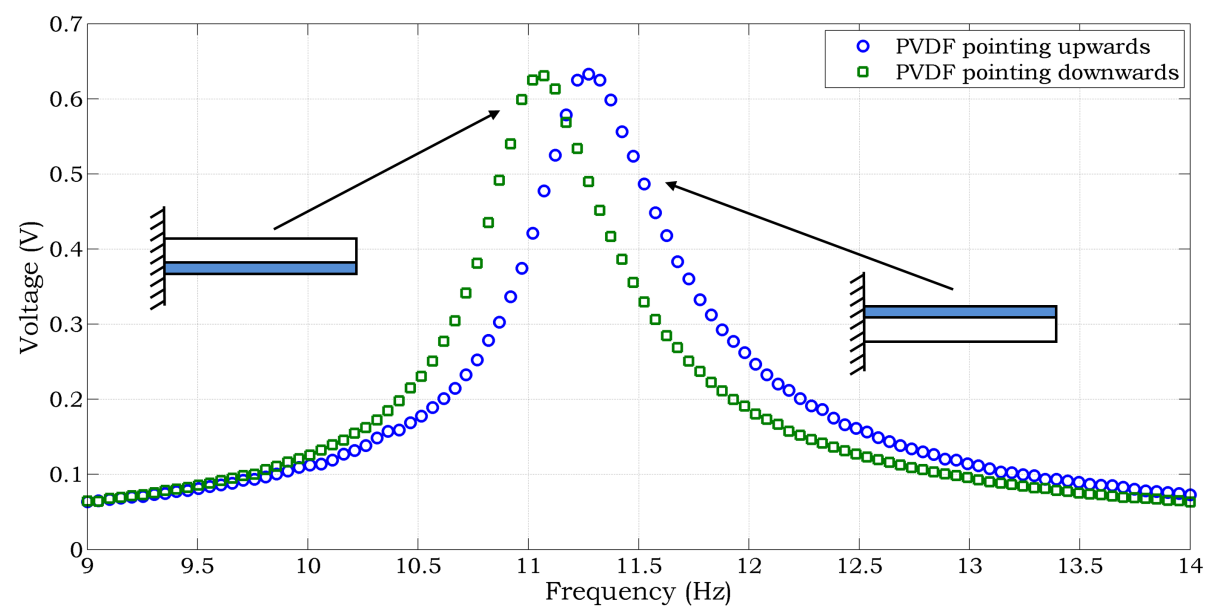

Figure 6: Experimental data from testing an energy harvester mounted horizontally with PVDF material as either the upper or lower layer of a unimorph. 

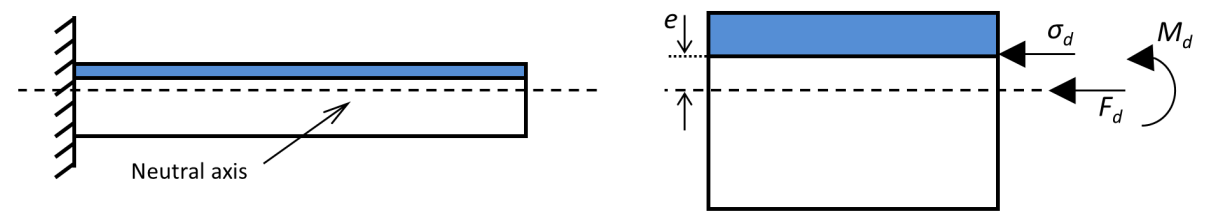

Figure 7: Schematic of the force and moment subjected to the beam from pre-stress in the adhesive layer.

Inclusion of pre-stress in the analytical model takes the form of an expression for additional potential strain energy. Potential energy from stresses and strains in a beam is given by:

$$
U_{d}=\frac{1}{2} \int_{x 1}^{x 2} \iint_{A}\left(\sigma_{11} \varepsilon_{11}\right) \mathrm{d} A \mathrm{~d} s .
$$

Note how only the region bound by $x_{1}$ and $x_{2}$ is of interest as this beam section contains piezoelectric material. Pre-stress occurring in the energy harvester can be represented as a force, $F_{d}$, and moment, $M_{d}=F_{d} e$, applied about the neutral axis of the device, see Figure 7. Here, $e$ refers to the distance between the neutral axis and the central reference axis along which pre-stress acts.

The pre-stress, $\sigma_{d}$, can be expressed by:

$$
\sigma_{11}=\sigma_{d}=\frac{F_{d}}{A_{d}}+\frac{F_{d} e y}{I_{c 1}}
$$

where $A_{d}$ is the cross-sectional area over which the pre-stress acts. For a unimorph harvester composition, $I_{c 1}$ is given by:

$$
I_{c 1}=I_{s 1}+I_{p 1}
$$

where the second moment of areas $I_{s 1}$ and $I_{p 1}$ are defined in Appendix A. Note how pre-stress is assumed to be uniform along the length of the beam. Although this was not confirmed experimentally, it is believed to be a valid assumption as the curing characteristics of the adhesive, and pre-stress in the PVDF strips during manufacture, is not expected to significantly change over a relatively short sample length.

Using Equation 24 in 23), and performing integration over the crosssectional area, yields:

$$
U_{d}=\frac{1}{2} \int_{x 1}^{x 2}\left(F_{d}+\frac{F_{d} e I_{c 2}}{I_{c 1}}\right)\left(v^{\prime}\right)^{2} \mathrm{~d} s,
$$

where constant $I_{c 2}$ for a unimorph composition is given by:

$$
I_{c 2}=b_{s} \frac{\left[\left(t_{s}-z\right)^{2}-(-z)^{2}\right]}{2}+b_{p} \frac{\left[\left(t_{s}+t_{p}-z\right)^{2}-\left(t_{s}-z\right)^{2}\right]}{2} .
$$


Adopting the procedure described in Section 2.2 on Equation (26), whereby Hamilton's principle and the calculus of variations is applied followed by the expression of $v$ in terms of normalised eigenvectors, $W_{r}(x)$, and time-dependant coordinates, $\eta_{r}(t)$, yields the following expression to be incorporated into Equation (22):

$$
U_{d}=\left(F_{d}+\frac{F_{d} e I_{c 2}}{I_{c 1}}\right) \eta(t) \int_{0}^{L} W^{\prime \prime}(s) W(s) \mathrm{d} s .
$$

In similar fashion to self-weight, the influence of pre-stress can be incorporated into the governing equations of motion to give:

$$
\begin{aligned}
\ddot{\eta}_{r}(t) & +2 \gamma_{r} \omega_{r} \dot{\eta}_{r}(t)+\left(\omega_{r}^{2}+\int_{0}^{L}\left(K_{8}(s) W^{\prime}(s)\right)^{\prime} W(s) \mathrm{d} s\right. \\
& \left.+\left(F_{d}+\frac{F_{d} e I_{c 2}}{I_{c 1}}\right) \int_{0}^{L} W^{\prime \prime}(s) W(s) \mathrm{d} s\right) \eta_{r}(t)+\left(C_{n 1}^{r}+C_{n 2}^{r}\right) \eta_{r}^{3}(t) \\
& +C_{n 3}^{r} \eta_{r}^{5}(t)+C_{n 4}^{r} \ddot{\eta}_{r}(t) \eta_{r}^{2}(t)+C_{n 5}^{r} \dot{\eta}_{r}^{2}(t) \eta_{r}(t)-C_{n 6}^{r} V(t)-C_{n 7}^{r} \eta_{r}(t) V(t) \\
& +C_{n 8}^{r} \eta_{r}^{2}(t) V(t)-C_{n 9}^{r} \eta_{r}^{3}(t) V(t)=C_{n 10}^{r} \ddot{w}_{b}(t)+\int_{0}^{L} K_{7}(s) W(s) \mathrm{d} s .
\end{aligned}
$$

This equation indicates that pre-stress influences the linear stiffness of the device.

\section{Further experimental work and model validation}

\subsection{Obtaining material properties}

Due to large variations in Young's moduli quoted by manufacturers, the determination of $E_{p}$ was first required. Static testing is used as a simple yet effective method to determine the material Young's modulus. For a cantilevered beam, Equation 30 provides the static tip deflection, $d$, as a result of self-weight

and pre-stress. The first term in this equation represents maximum deflection due to distributed force loading, and the second term represents maximum deflection resulting from an applied moment:

$$
d_{1,2}=\frac{F_{g} L_{p}^{4}}{8 E_{p} I_{p}} \pm \frac{M_{d} L_{p}^{2}}{2 E_{p} I_{p}}
$$

where the sign convention depends on sample mounting orientation. To expand, the deformation due to pre-stress either amplifies the deflection from self-weight (+ve) or reduces the deflection from self-weight (-ve). The two unknowns in Equation 30 are the Young's modulus, $E_{p}$, and moment, $M_{d}$. Expanding 
Equation (30) provides the following equations which can be solved simultaneously to estimate $E_{p}$ and $M_{d}$ :

$$
\begin{aligned}
& {\left[8 d_{1} \frac{b_{p} t_{p}^{3}}{12}\right] E_{p}+\left[4 L_{p}^{2}\right] M_{d}=\rho_{p} b_{p} t_{p} g L_{p}^{4},} \\
& {\left[8 d_{2} \frac{b_{p} t_{p}^{3}}{12}\right] E_{p}-\left[4 L_{p}^{2}\right] M_{d}=\rho_{p} b_{p} t_{p} g L_{p}^{4},}
\end{aligned}
$$

A PVDF sample with width $5 \mathrm{~mm}$ and thickness $0.08 \mathrm{~mm}$ was mounted at three overhang lengths of $70 \pm 1 \mathrm{~mm}, 56 \pm 1 \mathrm{~mm}$ and $34 \pm 1 \mathrm{~mm}$, with static deflections providing a Young's modulus, $E_{p}$, of $3.36 \mathrm{GPa}, 3.29 \mathrm{GPa}$ and $3.51 \mathrm{GPa}$, respectively. The same test was performed on several samples resulting in an average Young's modulus of $3.44 \mathrm{GPa}$; this magnitude will be utilised in any subsequent analytical model calculations.

The magnitude of the non-linear material constants, $\mu_{s 1}, \mu_{s 2}, \mu_{p 1}$ and $\mu_{p 2}$ also required prediction. Dynamic testing, in accordance with the procedure found in [10, was used to achieve this. Table 1 summarises the material properties obtained which will be used later in the analytical model.

Table 1: Material properties of FPEDs to use in analytical simulation; found from experimental testing (donated by ${ }^{*}$ ) and manufacturer data sheets.

\begin{tabular}{lll}
\hline \hline Parameter & Magnitude & \\
\hline & $\mathrm{Si}$ & $\mathrm{PVDF}$ \\
\hline Young's modulus, $E(\mathrm{~Pa})$ & $3.1 \times 10^{6}$ & $3.44 \times 10^{9 *}$ \\
Density, $\rho\left(\mathrm{kg} \cdot \mathrm{m}^{-3}\right)$ & 1250 & 1780 \\
Non-linear constant $\mu_{i 1}(\mathrm{~Pa})$ & $-1.5 \times 10^{9} *$ & $5 \times 10^{12 *}$ \\
Non-linear constant $\mu_{i 2}(\mathrm{~Pa})$ & $-1 \times 10^{15 *}$ & $3 \times 10^{21 *}$ \\
\hline Piezoelectric constant, $d_{31}\left(\mathrm{~m} . \mathrm{V}^{-1}\right)$ & $\mathrm{N} / \mathrm{A}$ & $15 \times 10^{-12}$ \\
Permittivity, $\varepsilon_{33}^{S}\left(\mathrm{~F} \cdot \mathrm{m}^{-1}\right)$ & $\mathrm{N} / \mathrm{A}$ & $6.55 \times 10^{-11}$ \\
\hline \hline
\end{tabular}

In the following two sections model validation of pre-stress and self-weight effects will be presented and discussed.

\subsection{Experiments regarding pre-stress}

In an attempt to minimise influences of pre-stress an alternate sample manufacturing procedure is proposed. Previously, during adhesive curing, samples were subjected to weight in-order to minimise the thickness of the adhesive layer. However, this is a major contributor to pre-stress due to the compressibility of the substrate Si material. Following the removal of weight after curing, relaxation of the $\mathrm{Si}$ induces stresses in addition to those present from adhesive shrinkage and PVDF material manufacture. Experimental data in Figure 8 is from samples manufactured without the added weight. 

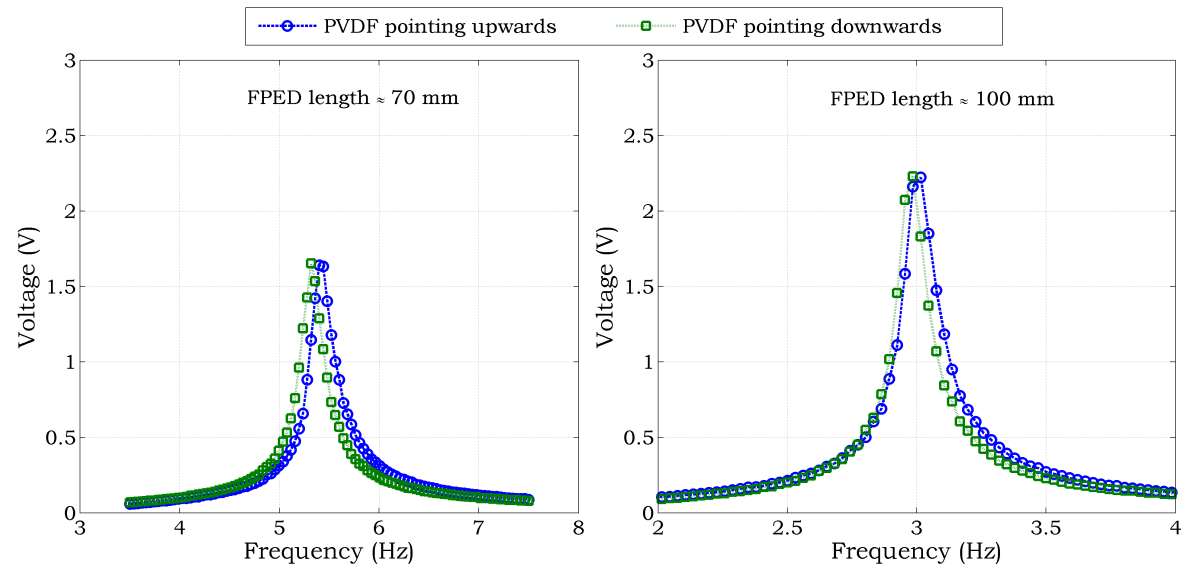

Figure 8: Experimental data from testing an energy harvester mounted horizontally with PVDF material as either the upper or lower layer. The added weight whilst adhesive curing takes place was removed during sample manufacture. Acc. level of $1 \mathrm{~ms}^{-2}$.

Figure 8 illustrates that reversing the orientation of a horizontally mounted sample, and therefore inherent pre-stress, has minimal effect on the dynamical behaviour of unimorph FPEDs manufactured without additional weight been used during curing. Percentage difference between the resonant frequencies whilst altering device orientation is $1.6 \%$ and $1 \%$ for devices of $70 \pm 1 \mathrm{~mm}$ and $100 \pm 1 \mathrm{~mm}$ in length, respectively. This example appears to negate the necessity of including pre-stress in the analytical model. However, referring back to Tang et al. 20, some bandwidth improving techniques rely on pre-stress as a means of resonant frequency control and the model presented in Section 2.3, with material and geometric non-linearity included, will be advantageous for future behavioural prediction of such configurations.

\subsection{Experiments regarding self-weight}

Attention is now diverted from pre-stress to self-weight. In this section validation of the self-weight model extension will be detailed along with a theoretical study examining the influence of self-weight on energy harvesting.

\subsubsection{Isolating self-weight effects}

Figure 9 shows frequency response data for an energy harvester in four orientations. Results from the developed analytical model are superimposed here for model validation purposes. The device had the following overhang dimensions:Si of $83 \pm 1 \times 10 \times 3.2 \mathrm{~mm}$ (including a $0.2 \mathrm{~mm}$ thick Si adhesive layer) and PVDF of $83 \pm 1 \times 10 \times 0.08 \mathrm{~mm}$. Curve fitting techniques on frequency response data revealed a mechanical damping ratio of approximately 0.02 for all cases with slight variations occurring as a result of clamping alterations during device reorientation. In order to minimise any non-linearity and concentrate efforts on 


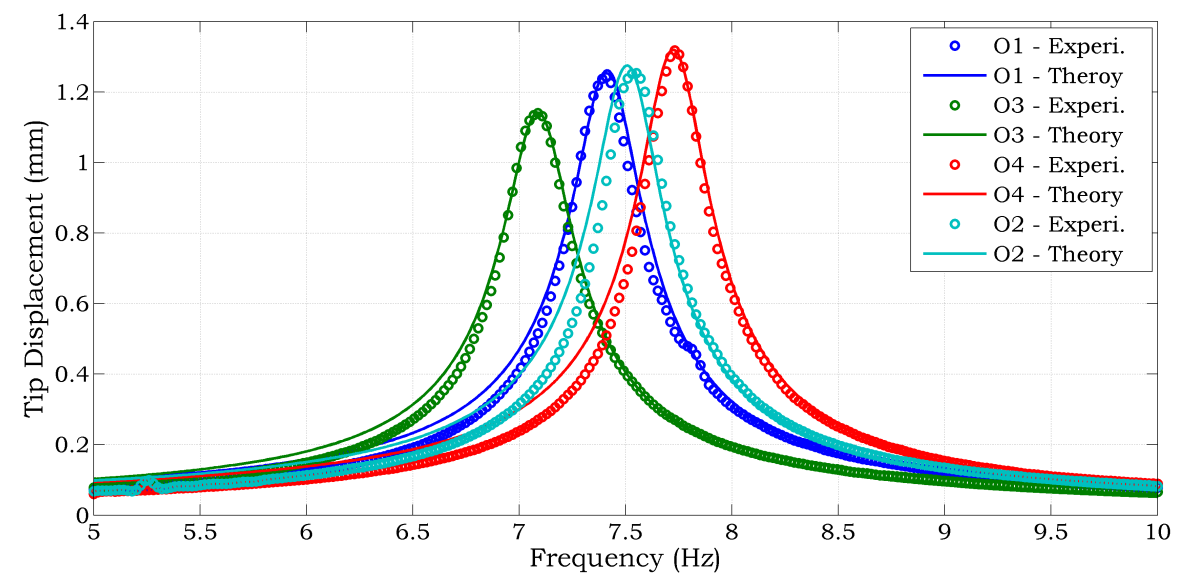

Figure 9: Experimental data from testing a FPED horizontally either with thickness parallel to the floor $(\mathrm{O} 1)$ or perpendicular to floor $(\mathrm{O} 2)$, and vertically, pointing either towards the ceiling (O3) or floor (O4).

establishing self-weight effects alone, the acceleration level applied during this set of experiments was $0.1 \mathrm{~ms}^{-2}$.

Figure 9 illustrates that the analytical model is capable of accurately predicting the effects of self-weight in terms of dynamical behaviour and energy harvester displacement. Experimental data indicates a percentage shift in resonant frequency between the two vertical configurations (O3 and O4) of 9.1\%, compared to $8.9 \%$ predicted by the theoretical model. In terms of individual orientations, regarding resonant frequency, a maximum difference of $2.6 \%$ between experimental data and theoretical predictions was obtained; this occurred for a beam oriented at $0^{\circ}(\mathrm{O} 2)$ with reasoning provided in Section 3.3 .3

\subsubsection{Initial tension due to self-weight}

Recall the model presented in Section 2.2 included an additional term representing the initial tension due to self-weight. In the previous example, where device length was approximately $83 \mathrm{~mm}$, this term had little influence on vibratory dynamics. However, if a device of significantly longer length, i.e. $152 \pm 1 \mathrm{~mm}$, was to be considered, the importance of this additional tension term becomes evident, see Figure 10.

Figure 10 shows the effects of modifying the TM and including tension due to initial deflection. The case with only TM modification (TM with distributed loading) produces a resonant frequency at $2.29 \mathrm{~Hz}$, which is a shift of $1.6 \%$ from the no gravity case (O1). However, if initial tension is also included, a resonant frequency of $2.56 \mathrm{~Hz}$ is obtained; $13 \%$ shift from O1. An experiment was conducted to verify such trends and results from testing a device with an overhang length of $153 \pm 1 \mathrm{~mm}$ are presented in Figure 11. Limitation in material availability imposed that the device had to be manufactured from two 


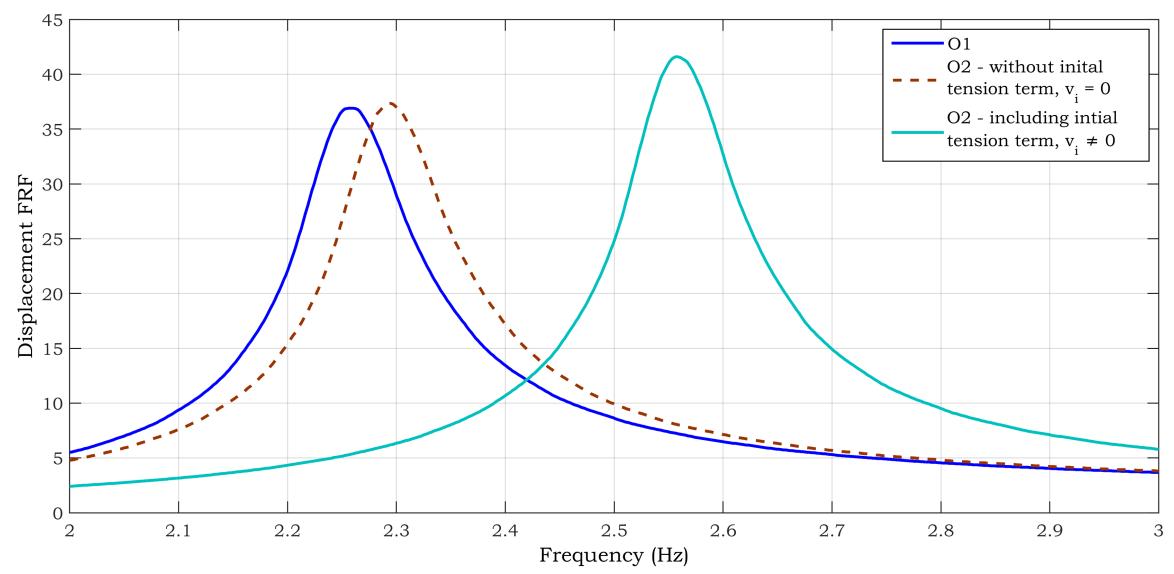

Figure 10: Theoretical displacement FRFs for a $150 \mathrm{~mm}$ FPED, where $\mathrm{O} 1$ and $\mathrm{O} 2$ orientations previously defined.

half-length PVDF layers adhered to a single long Si substrate. Charge was therefore only collected from the half closest to the clamp.

The importance of including tension from initial deformation is evident from the comparisons of Figures 10 and 11 . In the experiment, a shift of $20 \%$ in resonant frequency is observed when comparing the two horizontal beam cases. It is also interesting to observe the large differences in quality factor experienced by a device in these two orientations. Using the half power bandwidth method, overall damping for the device in orientation $\mathrm{O} 1$ is measured at 0.025 . In contrast, damping for the device orientated in $\mathrm{O} 2$ is measured at 0.016 . This $56 \%$ difference in damping can be used to justify the large differences in peak voltage and hence FPED performance and efficiency. It is thought to be attributed to differences in stress distribution at the clamp, i.e. support loss [22], between the two orientations as a result of significant self-weight.

It is worth mentioning that from this point forth only voltage FRFs will be generated and presented. This avoids unnecessary complications arising from using the vibrometer to measure potentially large deflections on materials with low surface roughness and high reflectiveness. Frequent reorientation of the vibrometer when testing at a range of mounting angles also deems use of the laser vibrometer impractical. Measuring and simulating voltage levels generated by FPEDs is therefore the recommended approach.

\subsubsection{Increasing acceleration}

In Section 3.3.1 the model was validated using low base acceleration levels to minimise non-linear effects. In this section, the validation is extended to a larger range of accelerations, inducing material and geometric non-linearity. The material non-linear coefficients reported in Table 1 are used in the simulations.

Figure 12 shows softening behaviour when the harvester is subjected to increasing levels of base excitation. This causes both reductions in resonant fre- 


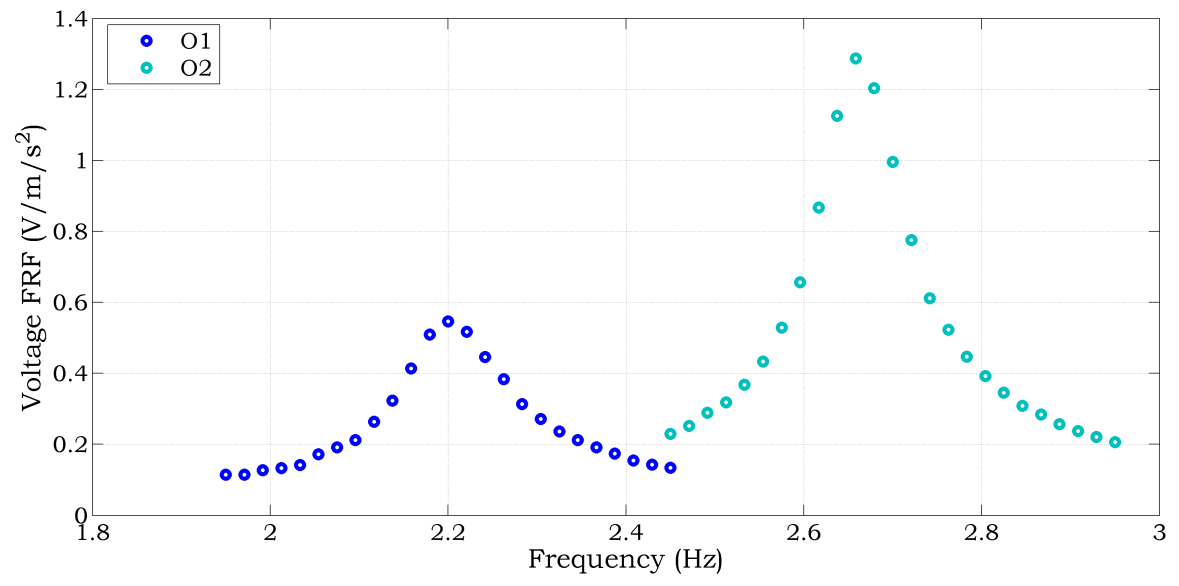

Figure 11: Voltage FRFs from experimental data for a FPED, where $\mathrm{O} 1$ and $\mathrm{O} 2$ orientations previously defined.

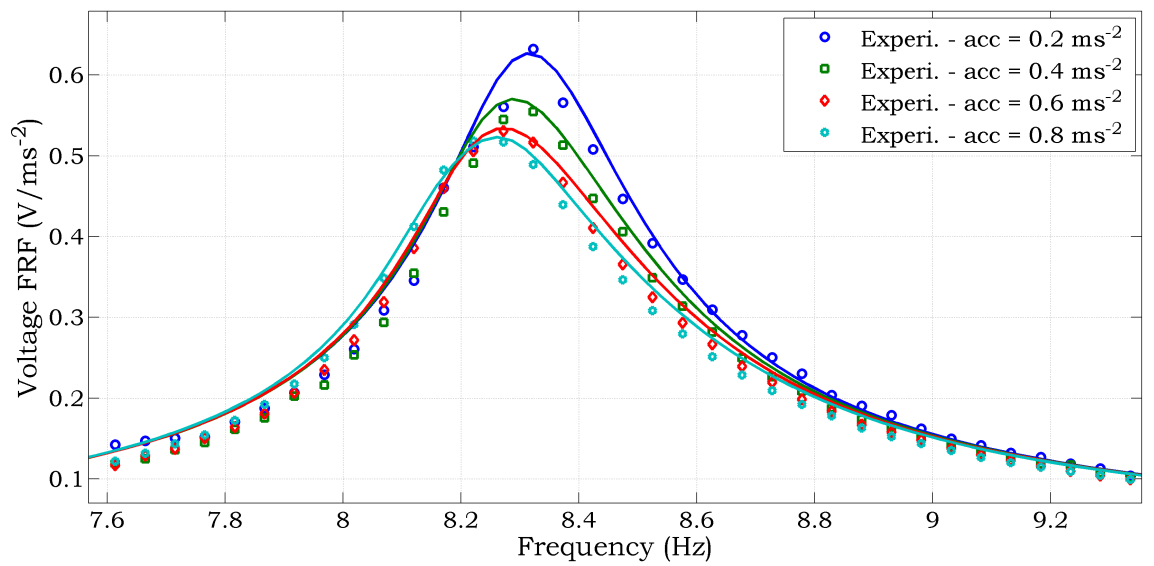

Figure 12: Experiment-theory comparisons of voltage FRFs for a FPED subjected to increasing excitation levels. Device oriented at $0^{\circ}$ with thickness direction perpendicular to floor. Solid lines represent simulated behaviour. Sample overhang length $=78.5 \pm 1 \mathrm{~mm}$. 

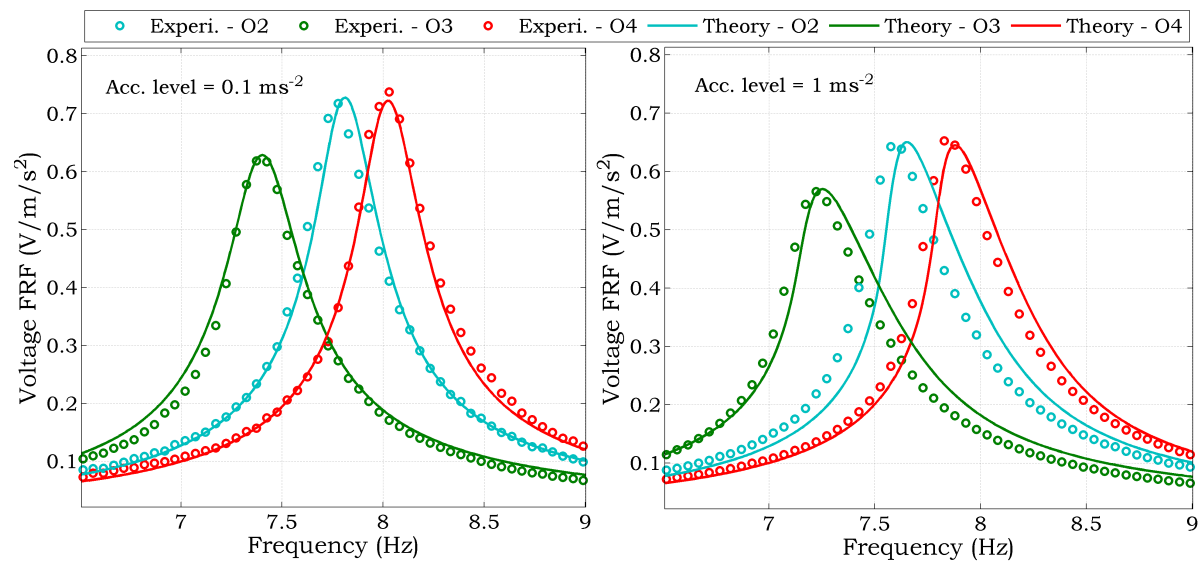

Figure 13: Experiment-theory comparisons of voltage FRFs for a FPED in various orientations, subjected to differing levels of acceleration.

quency and increases to mechanical damping. Phenomena by which damping changes with acceleration magnitude have previously been discussed in Nouira et al. [23. Here, curve fitting was used to estimate the damping ratio for each acceleration level with the following numerical information extracted: $\gamma=0.02$ at $0.2 \mathrm{~ms}^{-2}, \gamma=0.022$ at $0.4 \mathrm{~ms}^{-2}, \gamma=0.0235$ at $0.6 \mathrm{~ms}^{-2}$, and $\gamma=0.024$ at $0.8 \mathrm{~ms}^{-2}$. The results in Figure 12 indicate the model is capable of predicting the frequency location of maxima.

In Figure 13 the experimental-theoretical comparisons of an energy harvester in horizontal $(\mathrm{O} 2),+90^{\circ}(\mathrm{O} 3)$ and $-90^{\circ}(\mathrm{O} 4)$ orientations at acceleration levels of $0.1 \mathrm{~ms}^{-2}$ and $1 \mathrm{~ms}^{-2}$ are shown. The sample has an overhang length of $81 \pm 1 \mathrm{~mm}$, with remaining dimensions identical to those stated in Section 3.3.1. The results again highlight the validity of the model to predict resonant frequency locations at various acceleration levels. It is also evident that when peaks are not accurately located at low acceleration levels, discrepancies at resonant curve tails for higher acceleration levels are magnified. In this example, the resonant frequency of $\mathrm{O} 2$ was over-predicted at an excitation of $0.1 \mathrm{~ms}^{-2}$, with the most probable cause being inaccurate initial curvature estimations. To expand: clamping on two materials with vastly differing stiffness, in conjunction with the compressibility of the Si substrate, causes axial tension at the device root. This acts to alter the initial curvature of devices and hence the experimental data shows a marginally reduced resonant frequency in comparison to that predicted by the model.

Note for this particular device, voltage FRF peaks reduce by approximately $10 \%$ when base acceleration is increased from $0.1 \mathrm{~ms}^{-2}$ to $1 \mathrm{~ms}^{-2}$. Devices targeted at environments which induce non-linear behaviour through increased acceleration levels will therefore suffer from reduced efficiencies. 


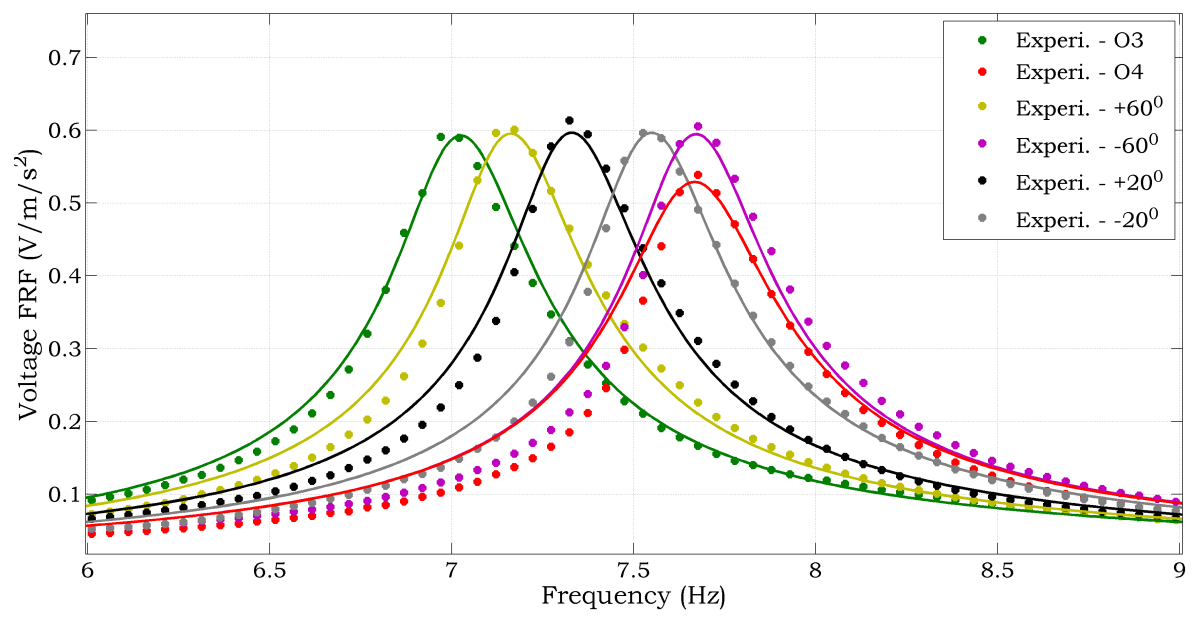

Figure 14: Experiment-theory comparisons of voltage FRFs for a FPED in intermediate orientation angles. $\mathrm{O} 3$ and $\mathrm{O} 4$ refer to the $+90^{\circ}$ and $-90^{\circ}$ orientations, respectively

\subsubsection{Intermediate orientation angles}

This final section presents full model validation by testing and simulating behaviour of FPEDs at various orientation angles between the two vertical extremes. Experimental data was acquired for a device with an overhang length of $83 \pm 1 \mathrm{~mm}$ oriented at angles of $\pm 90^{\circ}, \pm 60^{\circ}$ and $\pm 20^{\circ}$. An acceleration level of $0.2 \mathrm{~ms}^{-2}$ was applied in all cases. The outcome of testing along with the superposition of theoretical trends can be found in Figure 14

Several interesting observations can be made from experimental data alone. Firstly, peak voltages are not affected by FPED orientation about the z-axis; note the anomaly for $-90^{\circ}$ which resulted from clamp adjustment and so influenced mechanical damping. Secondly, the resonant frequency of a device oriented at $-60^{\circ}$ is similar to mounting at $-90^{\circ}$. At $-90^{\circ}$ the initial curvature is zero but the gravity force induces maximum tension in the device. However, at say $-60^{\circ}$, it is hypothesised that although induced tension resulting from the axial component of gravity (Equation (15p) has now reduced, the initial curvature at this orientation angle gives rise to additional tension (Equation (17)), resulting in a similar resonant frequency. As shown in Figure 14, theoretical findings also exhibit this behaviour. In-fact the model is reliable at predicting resonant frequencies at all orientation angles. Note how, in the theoretical model, damping magnitude had to be increased from 0.025 (all cases) to 0.028 when fitting device behaviour at $-90^{\circ}$.

The analytical model is now used to gauge the effects of device mounting angle on the resonant frequency of a FPED. Figure 15 provides theoretical trends between orientation angle and resonant frequency for two harvesters subjected to a $0.1 \mathrm{~ms}^{-2}$ acceleration. Mechanical damping in both cases is assumed constant at 0.025. For reference, the un-damped fundamental frequencies are also 

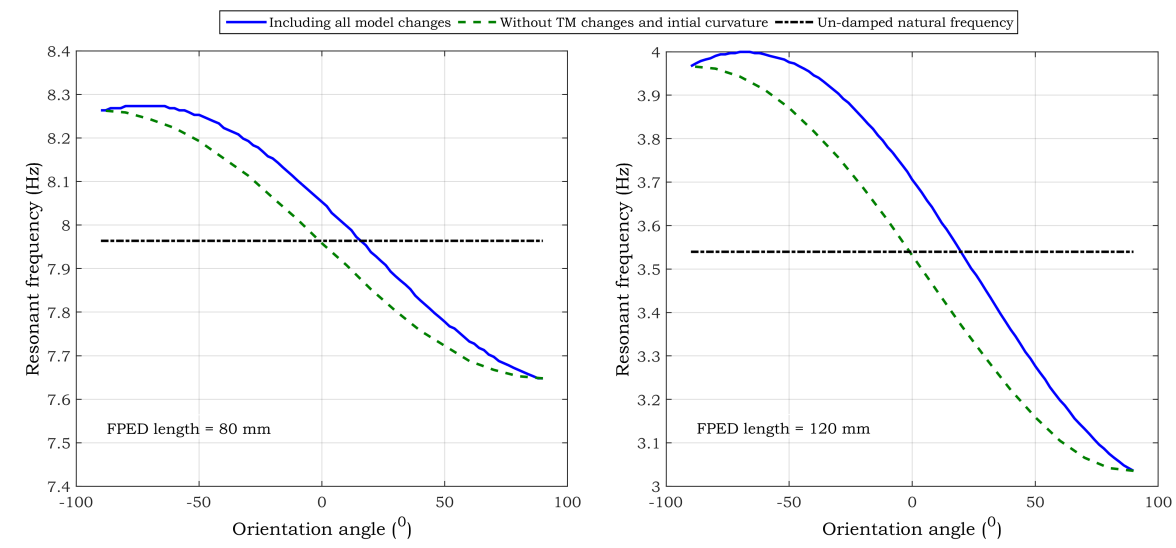

Figure 15: Theoretical predictions of FPED resonant frequency across a range of mounting angles whilst isolating various model contributions.

provided in Figure 15.

Resonant frequency generally reduces as $\theta$ is increased from $-90^{\circ}$ to $90^{\circ}$. As a result of tension from initial static deformation and TM modification, a non-sinusoidal relationship was found. If these model additions are omitted from theoretical calculation a sinusoidal relationship is obtained, see Figure 15 However, as discovered earlier in Figure 14, this sinusoidal relationship is not supported through experimental testing. The percentage difference between the two extreme resonant frequencies for harvesters of $80 \mathrm{~mm}$ and $120 \mathrm{~mm}$ in length was $8 \%$ and a substantial $34 \%$ respectively. This example highlights the importance of correctly modelling the influences of self-weight on FPEDs. Without accurate knowledge of resonant frequency, performance predictions of FPEDs cannot be made with confidence. Therefore the modelling extensions presented in Section 2.2 are highly recommended when the situation necessitates them.

\section{Conclusions}

This paper has presented key modelling extensions and improvements to an existing non-linear analytical model, with validation provided through extensive experimental testing. Focus was on the incorporation of effects expected to affect the dynamical behaviour of highly flexible piezoelectric devices (FPEDs), and currently these include higher orders of material non-linearity, self-weight and pre-stress. Pre-stress can result from both curvature of the piezoelectric film during manufacture and state change of the adhesive during curing. Incorporation was in the form of stress along the bonding layer of devices resulting in an additional potential energy term. However, it was shown that careful manufacture of samples can alleviate the need for including pre-stress in the theoretical model. 
Modelling the influences of self-weight required (i) modification of the transfer matrices and (ii) derivation of potential energy terms. It was found that incorporating initial curvature, hence some tension, was hugely important to replicate behaviour observed during experimental testing.

Testing was performed in a manner which isolated various aspects of the model. Lower acceleration levels minimised non-linear effects such that influences of self-weight dominated. Acceleration levels were later increased to those inducing non-linear behaviour. Although the majority of testing was performed with devices mounted such that their thickness direction was perpendicular to the floor, devices were also mounted with their thickness direction parallel to the floor to minimise self-weight effects and provide a 'control' case. Various mounting angles were also examined to gauge the accuracy of developed models. Mechanical properties for the PVDF were obtained through static testing whereas non-linear coefficients for both substrate and piezoelectric materials were obtained through dynamic testing and curve fitting.

Experiments reveal that the addition of self-weight to the model is crucial in predicting the resonant frequency and therefore performance of FPEDs. In one case the percentage difference between resonant frequency extremes was over $30 \%$. It was interesting to discover that the relationship between mounting angle and resonant frequency is not sinusoidal for devices of high flexibility. This resulted in observations such as:- a device mounted at either $-60^{\circ}$ or $-90^{\circ}$ has a similar resonant frequency. It is also worth noting that although resonant frequencies were found to be sensitive to mounting angle about the z-axis, the peak voltages were largely uninfluenced. However, for the longer devices it was observed that severe reductions in voltage generation and efficiency result from mounting devices horizontally with their thickness direction perpendicular to the direction of gravity.

Capabilities of the presented model whilst incorporating non-linear effects in addition to self-weight were also highlighted. In all cases the shift in resonance was correctly predicted to within $1 \%$ and mounting angle was found to have little effect towards the extent of non-linearity. As is known in the field, and here confirmed for FPEDs, material non-linearities dominate those from the geometry causing reduction in resonant frequency with increasing levels of acceleration. Overall, the ability of the developed model to predict general behavioural trends is clear from this paper.

\section{Acknowledgements}

This research was partially supported by the International Exchanges Scheme of the Royal Society of London, U.K..

\section{References}

[1] S. Roundy, E. S. Leland, J. Baker, E. Carleton, E. Reilly, E. Lai, B. Otis, J. M. Rabaey, P. K. Wright, V. Sundararajan, Improving power output for vibration-based energy scavengers, Journal of Pervasive Computing, IEEE 4 (2005) 28-36. 
[2] H. Mutsuda, R. Watanabe, S. Azuma, Y. Tanaka, Y. Doi, Ocean power generator using flexible piezoelectric device, 32nd International Conference on Ocean, Offshore and Arctic Engineering, 9-14th June 2013, Nantes, France, 2013, pp. V008T09A002 - 9 pages.

[3] Y. Tanaka, T. Oko, H. Mutsuda, A. A. Popov, R. Patel, S. McWilliam, Forced vibration experiments on flexible piezoelectric devices operating in air and water environments, International Journal of Applied Electromagnetics and Mechanics 45 (2013) 573-580.

[4] H. A. Sodano, G. Park, D. J. Inman, Estimation of electric charge output for piezoelectric energy harvesting, Strain 40 (2004) 49-58.

[5] A. Erturk, D. J. Inman, A distribution parameter electromechanical model for cantilever piezoelectric energy harvesters, Journal of Sound and Acoustics 130 (2008) 041002 (15pp).

[6] R. Patel, S. McWilliam, A. A. Popov, A geometric parameter study of piezoelectric coverage on a rectangular cantilever energy harvester, Smart Materials and Structures 20 (2011) 085004 (12pp).

[7] S. C. Stanton, A. Erturk, B. P. Mann, D. J. Inman, Nonlinear piezoelectricity in electroelastic energy harvesters: Modeling and experimental identification, Journal of Applied Physics 108 (2010) 074903 (9pp).

[8] K. H. Mak, A. A. Popov, S. McWilliam, Experimental model validation for a nonlinear energy harvester incorporating a bump stop, Journal of Sound and Vibration 331 (2012) 2602-2623.

[9] S. C. Stanton, A. Erturk, B. P. Mann, E. H. Dowell, D. J. Inman, Nonlinear nonconservative behavior and modeling of piezoelectric energy harvesters including proof mass effects, Journal of Intelligent Material Systems and Structures 23 (2012) 183-199.

[10] R. Patel, S. McWilliam, A. A. Popov, Optimization of piezoelectric cantilever energy harvesters including non-linear effects, Smart Materials and Structures 23 (2014) 085002 (17pp).

[11] S. P. Joshi, Non-linear constitutive relations for piezoceramic materials, Smart Materials and Structures 1 (1992) 80-83.

[12] L. N. Virgin, S. T. Santillan, D. B. Holland, Effect of gravity on the vibration of vertical cantilevers, Mechanics Research Communications 34 (2007) $312-317$.

[13] J. D. Hobeck, D. J. Inman, Artificial piezoelectric grass for energy harvesting from turbulence-induced vibration, Smart Materials and Structures 21 (2012) 105024 (10pp). 
[14] X. D. Xie, N. Wu, K. V. Yuen, Q. Wang, Energy harvesting from high-rise buildings by a piezoelectric coupled cantilever with a proof mass, International Journal of Engineering Science 72 (2013) 98-106.

[15] M. Friswell, S. Ali, O. Bilgen, S. Adhikari, A. Lees, G. Litak, Non-linear piezoelectric vibration energy harvesting from a vertical cantilever beam with tip mass, Journal of Intelligent Material Systems and Structures 23 (2012) 1505-1521.

[16] S. R. Oh, K. Yao, C. L. Chow, F. E. H. Tay, Residual stress in piezoelectric poly(vinylidene-fluoride-co-trifluoroethylene) thin films deposited on silicon substrates, Thin Film Solids 519 (2010) 1441-1444.

[17] J. A. Nairn, Energy release rate analysis for adhesive and laminate double cantilever beam specimens emphasizing the effect of residual stresses, International Journal of Adhesion \& Adhesives 20 (1999) 59-70.

[18] R. Patel, Modelling analysis and optimisation of cantilever piezoelectric energy harvesters, Ph.D. thesis, University of Nottingham, University Park, Nottingham, UK (2012).

[19] MATHWORKS, website last accessed on 20-05-2015, URL:http://www . mathworks.co.uk/ (2015).

[20] L. Tang, Y. Yang, C. K. Soh, Toward broadband vibration-based energy harvesting, Journal of Intelligent Material Systems and Structures 21 (2010) 1867-1897.

[21] R. Tiwari, Analysis and Identification in Rotor-Bearing Systems, Indian Institute of Technology Guwahati, 2010.

[22] Z. Hao, A. Erbil, F. Ayazi, An analytical model for support loss in micromachined beam resonators with in-plane flexural vibrations, Sensors and Actuators, A: Physical 109 (2003) 156-164.

[23] H. Nouira, E. Foltête, L. Hirsinger, S. Ballandras, Investigation of the effects of air on the dynamic behavior of a small cantilever beam, Journal of Sound and Vibration 305 (2007) 243-260.

\section{Appendix A. Potential energy expression constants}

In this appendix, constants $K_{1}$ through to $K_{6}$ found in the potential energy expression, Equation (8), are defined. They utilise Heaviside step functions, $H$, to isolate various sections of the beam, i.e. those comprising of piezoelectric 
material and those with substrate only, and are given by:

$$
\begin{aligned}
K_{1}(s) & =\left(H(s-0)-H\left(s-x_{1}\right)\right) E_{s} I_{s}+\left(H\left(s-x_{1}\right)-H\left(s-x_{1}-x_{2}\right)\right) E_{s} I_{s 1} \\
& +\left(H\left(s-x_{1}\right)-H\left(s-x_{1}-x_{2}\right)\right) E_{p} I_{p 1}+\left(H\left(s-x_{1}-x_{2}\right)-H(s-L)\right) E_{s} I_{s}, \\
K_{2}(s)= & \left(H(s-0)-H\left(s-x_{1}\right)\right) \mu_{s 1} I_{s 4}+\left(H\left(s-x_{1}\right)-H\left(s-x_{1}-x_{2}\right)\right) \mu_{s 1} I_{s 2} \\
& \left(H\left(s-x_{1}\right)-H\left(s-x_{1}-x_{2}\right)\right) \mu_{p 1} I_{p 2}+\left(H\left(s-x_{1}-x_{2}\right)-H(s-L)\right) \mu_{s 1} I_{s 4}, \\
K_{3}(s)= & \left(H(s-0)-H\left(s-x_{1}\right)\right) \mu_{s 2} I_{s 5}+\left(H\left(s-x_{1}\right)-H\left(s-x_{1}-x_{2}\right)\right) \mu_{s 2} I_{s 3} \\
& \left(H\left(s-x_{1}\right)-H\left(s-x_{1}-x_{2}\right)\right) \mu_{p 2} I_{p 3}+\left(H\left(s-x_{1}-x_{2}\right)-H(s-L)\right) \mu_{s 2} I_{s 5}, \\
K_{4}(s)= & \left(H\left(s-x_{1}\right)-H\left(s-x_{1}-x_{2}\right)\right)\left(2 E_{p} d_{31} b_{p}\right)\left(t_{s}+\frac{t_{p}}{2}-\bar{y}\right), \\
K_{5}(s)= & \left(H\left(s-x_{1}\right)-H\left(s-x_{1}-x_{2}\right)\right)\left(\frac{\mu_{p 3} I_{p 1}}{t_{p}}\right), \\
K_{6}(s)= & \left(H\left(s-x_{1}\right)-H\left(s-x_{1}-x_{2}\right)\right)\left(\frac{b_{p} \varepsilon_{33}}{t_{p}}\right) .
\end{aligned}
$$

where $b$ and $t$ are the layer widths and thickness, and subscripts ' $p$ ' and ' $s$ ' refer to piezoelectric and substrate layers respectively. $\bar{y}$ is the location of the neutral axis from the bottom of the substrate layer, and geometry dependant $I_{s}, I_{s 1}, I_{s 2}, I_{s 3}, I_{s 4}, I_{s 5}, I_{p 1}, I_{p 2}$ and $I_{p 3}$ for a unimorph device composition are provided by:

$$
\begin{aligned}
I_{s} & =\frac{b_{s} t_{s}^{3}}{12} \\
I_{s 1} & =b_{s}\left[\left(t_{s}\right) \bar{y}^{2}-\left(t_{s}^{2}\right) \bar{y}+\frac{1}{3} t_{s}^{3}\right], \\
I_{s 2} & =b_{s}\left[\frac{\left(t_{s}-\bar{y}\right)^{5}}{5}-\frac{(-\bar{y})^{5}}{5}\right], \\
I_{s 3} & =b_{s}\left[\frac{\left(t_{s}-\bar{y}\right)^{7}}{7}-\frac{(-\bar{y})^{7}}{7}\right], \\
I_{s 4} & =\frac{b_{s} t_{s}^{5}}{80} \\
I_{s 5} & =\frac{b_{s} t_{s}^{7}}{320} \\
I_{p 1} & =b_{p}\left[\left(t_{p}\right) \bar{y}^{2}+\left(-2 t_{p} t_{s}-t_{p}^{2}\right) \bar{y}+\left(\frac{1}{3} t_{p}^{3}+t_{p}^{2} t_{s}+t_{p} t_{s}^{2}\right)\right], \\
I_{p 2} & =b_{p}\left[\frac{\left(t_{s}+t_{p}-\bar{y}\right)^{5}}{5}-\frac{\left(t_{s}-\bar{y}\right)^{5}}{5}\right],
\end{aligned}
$$




$$
I_{p 3}=b_{p}\left[\frac{\left(t_{s}+t_{p}-\bar{y}\right)^{7}}{7}-\frac{\left(t_{s}-\bar{y}\right)^{7}}{7}\right]
$$

\section{Appendix B. Time-independent constants}

In this appendix the time-independent constants, $C_{n 1}$ through to $C_{n 15}$, are defined. These constants can be found in the equations of motion, Equations $(10)$ and (11), as multipliers to powers and derivatives of the time-dependant variable, $\eta_{r}$.

$$
\begin{aligned}
C_{n 1}^{r}= & {\left[\int_{0}^{L} W_{q}(s)\left[2 K_{2}(s) W_{r}^{\prime \prime 3}(s)\right]^{\prime \prime} \mathrm{d} s\right] } \\
C_{n 2}^{r}= & {\left[\int_{0}^{L} W_{q}(s)\left[\left(K_{1}(s) W_{r}^{\prime \prime}(s) W_{r}^{\prime}(s)\right)^{\prime} W_{r}^{\prime}(s)\right]^{\prime} \mathrm{d} s\right] } \\
C_{n 3}^{r}= & {\left[\int_{0}^{L} W_{q}(s)\left[3 K_{3}(s) W_{r}^{\prime \prime 5}(s)\right]^{\prime \prime} \mathrm{d} s\right] } \\
C_{n 4}^{r}= & {\left[\int_{0}^{L} W_{q}(s)\left[W_{r}^{\prime}(s) \int_{L}^{s} m(s) \int_{0}^{s}\left(W_{r}^{\prime \prime}(s) W_{r}^{\prime}(s)\right) \mathrm{d} s \mathrm{~d} s\right]^{\prime} \mathrm{d} s\right] } \\
C_{n 5}^{r}= & {\left[\int_{0}^{L} W_{q}(s)\left[W_{r}^{\prime}(s) \int_{L}^{s} m(s) \int_{0}^{s}\left(W_{r}^{\prime 2}(s)\right) \mathrm{d} s \mathrm{~d} s\right]^{\prime} \mathrm{d} s\right] } \\
C_{n 6}^{r}= & {\left[\int_{0}^{L} W_{q}(s)\left[\frac{1}{2} K_{4}(s)\right]^{\prime \prime} \mathrm{d} s\right] V(t) } \\
C_{n 7}^{r}= & {\left[\int_{0}^{L} W_{q}(s)\left[\left(K_{5}(s)\right) W_{r}^{\prime \prime}(s)\right]^{\prime \prime} \mathrm{d} s\right] } \\
C_{n 8}^{r}= & {\left[\int_{0}^{L} W_{q}(s)\left[\frac{1}{2} K_{4}(s) W_{r}^{\prime \prime}(s) W_{r}^{\prime}(s)\right]^{\prime} \mathrm{d} s\right] } \\
C_{n 11}^{r}= & {\left.\left[\int_{0}^{L} K_{5}(s) W_{r}^{\prime \prime 2}(s) \mathrm{d} s\right] \int_{V_{p}}^{L} W_{q}(s)\left[\frac{1}{4} K_{4}(s) W_{r}^{\prime 2}(s)\right]^{\prime \prime} \mathrm{d} s\right] } \\
C_{n 12}^{r}= & {\left[\int_{0}^{L} 2 K_{5}(s)\left(W_{r}^{\prime \prime 2}(s) W_{r}^{\prime 2}(s)\right) \mathrm{d} s\right] } \\
C_{n 10}^{r}= & {\left[\int_{0}^{L} W_{q}(s)\left[\left(\left(K_{5}(s)\right) W_{r}^{\prime \prime}(s) W_{r}^{\prime}(s)\right)^{\prime} W_{r}^{\prime}(s)\right]^{\prime} \mathrm{d} s\right] }
\end{aligned}
$$




$$
\begin{aligned}
& C_{n 13}^{r}=\left[\int_{0}^{L} \frac{1}{2} K_{4}(s) W_{r}^{\prime \prime}(s) \mathrm{d} s\right] \\
& C_{n 14}^{r}=\left[\int_{0}^{L} \frac{3}{2} K_{4}(s)\left(\frac{1}{2} W_{r}^{\prime \prime}(s) W_{r}^{\prime 2}(s)\right) \mathrm{d} s\right] \\
& C_{n 15}^{r}=\left[\int_{0}^{L} K_{6}(s) \mathrm{d} s\right]
\end{aligned}
$$

In the above expressions, $W_{q}$ and $W_{r}$ are the mass normalised mode shapes of the $q^{\text {th }}$ and $r^{\text {th }}$ mode, respectively. Definitions of constants $K_{1}$ to $K_{6}$ can be found in Appendix A. 